\title{
Nuevas opciones de tratamiento para el paciente con COVID-19 La experiencia hematológica
}

Roberto Ovilla-Martínez * ${ }^{*}$ Pamela E. Báez-Islas ${ }^{1}$ Xóchitl Cota-Rangel' y Antonio de la Peña-Celaya1

Servicio de Hematología, Hospital Ángeles Lomas, Huixquilucan, Edo. Méx., México

\section{Resumen}

En la actual pandemia de enfermedad por coronavirus 2019 (COVID-19) se ha observado que las principales complicaciones se presentan como resultado de la liberación de múltiples citocinas como interleucina (IL) 1, IL-6, factor de necrosis tumoral alfa e interferones de tipo 1 que generan un estado proinflamatorio caracterizado por lesión tisular pulmonar y subsecuentemente falla orgánica múltiple. En el campo de la hematología se cuenta con experiencia en el uso de diversos fármacos diseñados para limitar estas citocinas los cuales se han utilizado ya en pacientes con COVID-19 entre los que se encuentran los inhibidores de la IL-6 como el tocilizumab, el sarilumab y el siltuximab, el inhibidor de IL-1 anakinra y los inhibidores de la janus cinasa ruxolitinib y baricitinib. Al conocer la base fisiopatológica de la COVID-19, la utilidad de este tipo de fármacos muestra resultados alentadores para los cuadros moderados a graves de la enfermedad y extender su uso en ensayos clínicos mayores.

PALABRAS CLAVE: COVID-19. Tocilizumab. Sarilumab. Siltuximab. Baricitinib. Ruxolitinib.

\section{New therapeutic options for the COVID-19 patient. The experience from the hematologist}

\begin{abstract}
In the current SARS-COV-2 pandemic, it has been observed that the main complications arise as a result of the release of multiple cytokines such as IL-1, IL-6, TNF- $\alpha$ and type 1 interferons that generate a proinflammatory state characterized by lung tissue injury and subsequently multiple organ failure. In the hematology field, there is experience in the use of various drugs designed to limit these cytokines which have already been used in patients with COVID-19 including IL-6 inhibitors such as tocilizumab, sarilumab, and siltuximab; the IL-1 inhibitor anakinra; and the janus kinase inhibitors ruxolitinib and baricitinib. Knowing the pathophysiological basis of COVID-19, the usefulness of this type of drugs show encouraging results for moderate to severe symptoms of the disease and encourages its use in larger clinical trials.
\end{abstract}

KEY WORDS: COVID-19. Tocilizumab. Sarilumab. Siltuximab. Baricitinib. Ruxolitinib.

\section{Introducción}

Como es bien sabido, el nuevo coronavirus, nombrado en febrero 2020 por la Organización Mundial de la Salud (OMS) coronavirus 2 del síndrome respiratorio agudo grave (SARS-CoV-2), genera la enfermedad por coronavirus 2019 (COVID19). Pandemia que hasta el día 21 de junio contaba con $8,690,140$ de casos confirmados por la OMS, con 461,274 defunciones ${ }^{1,2}$. En México, según el informe 
nacional por la Secretaría de Salud del día 6 de diciembre del 2021 se han informado de 3,901 263 casos con 295,203 defunciones $^{3}$. Si bien la principal afectación de esta enfermedad es en vías aéreas bajas por su mayor severidad manifestada por el síndrome de insuficiencia respiratoria, desde la perspectiva de la hematología se han observado diversas alteraciones propias de esta especialidad, como son las diversas alteraciones en hemostasia y trombosis, las cuales se abordarán en otra revisión, y la desregulación del sistema inmunitario, que ha dado la pauta para tratamientos de amplio uso en el contexto de la hematología.

Una manera en que se ha clasificado la COVID-19 es con base en la fase viral o inmunitaria en la que se encuentra el paciente. La primera fase es la viral, caracterizada por fiebre, malestar general y linfopenia. Posteriormente le sigue la fase de respuesta inmunitaria del huésped, donde si esta es excesiva, genera inflamación sistémica que caracteriza el cuadro de neumonía grave, insuficiencia cardiaca, choque séptico resultado de la disminución en células $T$ $\mathrm{CD} 4+\mathrm{y} T$ reguladoras (Treg) con incremento de citocinas y biomarcadores como interleucina (IL) 2, IL-6, IL-7, factor estimulante de colonias de granulocitos (GCSF), proteína inflamatoria de macrófagos 1- $\alpha$, factor de necrosis tumoral (TNF), proteína $C$ reactiva (PCR), ferritina y dímero $D^{4}$.

La transmisión se da humano a humano. La proteína $S$ del coronavirus se une a las células huésped mediante el receptor de la enzima convertidora de angiotensina 2 (ACE2) fusionándose con la membrana celular y liberando el ARN viral. A nivel intracelular, el ARN es identificado y usualmente, mediante receptores tipo toll $3,7,8$ y 9 , es reconocido en el endosoma. El receptor de ARN tipo gen inducible por acido retinoico, receptor de melanoma citosólico asociado a diferenciación tipo 5 y nucleotidiltransferasa cíclica GMP-AMP sintetasa son los responsables del reconocimiento de ARN viral en el citoplasma. Estos complejos celulares generan diversas cascadas de señalización que activan factores de transcripción nuclear tipo factor-KB y factor regulatorio de interferón con la producción de interferones tipo I (IFN- $\alpha / \beta$ ) y una serie de citocinas proinflamatorias ${ }^{1,5}$. Siendo así, los interferones tipo I (IFN-I) mantienen un rol vital en la estimulación de macrófagos-monocitos y células dendríticas con producción de IL-6, TNF- $\alpha$ e IL1- $\beta$, citocinas que ya se han visto elevadas en las formas severas de SARS-CoV- $2^{6,7}$. Varias de estas citocinas estimulan a los linfocitos NK y linfocitos $T$
(CD8+, CD4+, Treg) provocando una segunda ola de citocinas y generando lo que se conoce como síndrome de liberación de citocinas o tormenta de citocinas $^{6,8,9}$. Estas citocinas generan una importante respuesta inflamatoria en las vías aéreas inferiores con subsecuente lesión pulmonar. Así pues, la tormenta de citocinas liberadas en pacientes con COVID19 causada primero por la invasión de la mucosa respiratoria y después por la respuesta inmunitaria desmedida es la causa de la condición crítica de los pacientes con COVID-19.

En la experiencia clínica reportada, se han observado diversas citocinas elevadas en pacientes con COVID-19: IL-1, IL-2, IL-4, IL-6, IL-7, IL-10, IL-12, IL-13, IL-17, GCSF, factor estimulante de colonias de monocitos, proteína inducida 10, proteína quimioatrayente de monocitos 1 (MCP-1), proteína inflamatoria de los macrófagos $1 \alpha$ (MIP-1 $\alpha$ ), factor de crecimiento de hepatocitos, interferón gamma (IFN- $\gamma$ ) y TNF- $\alpha^{1,5}$. Otro estudio en los primeros grupos de población china afectada con neumonía severa demostró que los principales valores de laboratorio alterados fueron linfopenia, elevación de PCR con procalcitonina normal y elevación de deshidrogenasa láctica (DHL) con disminución de albúmina. Al medir niveles de diversas citocinas observaron que existían diferencias significativas entre la expresión de receptor de interleucina 2 (IL-2R) y los niveles séricos de IL-6 al compararlo en tres grupos según la gravedad de la infección. Se observó que estas eran mayores en el grupo crítico comparado con el grave y mayores en el grupo grave comparado con afectación media. No se encontraron diferencias significativas entre los niveles séricos de TNF- $\alpha$, IL-1, IL-8, IL-10, PCR de alta sensibilidad ni conteo de linfocitos o DHL entre estos tres grupos $(p>0.05)^{10}$.

Otro dato relevante es que los IFN-I también regulan a la alza la expresión de los receptores de la ACE2, el cual es también receptor para SARS-CoV-2 ${ }^{11}$. También es sabido que en diversas infecciones, tanto virales como bacterianas, los IFN-I juegan un papel primordial en la sobreactivación del sistema de coagulación desarrollando coagulación intravascular diseminada ${ }^{9}$. El modelo de afectación de IFN-I se ha replicado en modelos murinos infectados con SARSCoV, donde se observó que aquellos ratones deprivados de IFN-I mostraban menor tasa de letalidad comparado con los ratones wild-type. Esta información es relevante porque la señalización celular de los IFN-I se realiza a través de la membrana celular por receptores IFN- $\alpha / \beta$ que usan la vía de JAK/STAT ${ }^{8,12}$. 
Con estos datos podemos concluir que una de las principales acciones para evitar desenlaces fatales en pacientes afectados con COVID-19 es buscar la limitación de la respuesta inmunitaria proinflamatoria, teniendo así una amplia opción de terapias, como se mencionó anteriormente.

\section{Inhibidores de la interleucina 6}

Diversos centros de hospitalización han sugerido el uso del inhibidores de IL-6 como el tocilizumab, el sarilumab y el siltuximab, siendo el de mayor uso hasta ahora el tocilizumab, con registro hasta el momento de 57 estudios clínicos en clinicaltrials.gov con dosis desde 400 hasta $1,200 \mathrm{mg}^{13-15}$. El primer reporte de efectividad con tocilizumab fue el de los resultados preclínicos en China, donde se observó que el $75 \%$ de un grupo de 20 pacientes tratados con tocilizumab mostraron mejoría en parámetros de oxigenación ${ }^{16}$. La mayor serie reportada hasta el momento son los 100 pacientes italianos con SARSCoV-2 y requerimiento de oxígeno suplementario en Brescia (Italia), donde se utilizó tocilizumab a dosis de $8 \mathrm{mg} / \mathrm{kg}$ cada 12 horas por 2 dosis. Al inicio del tratamiento se refiere que 43 pacientes se encontraban en la terapia intensiva. De los 57 pacientes fuera de unidad de terapia intensiva (UTI), el 65\% mejoraron con suspensión de la ventilación no invasiva, el $12 \%$ se mantuvo estable y el $23 \%$ fallaron al tratamiento con tres ingresos a UTI y 10 defunciones. En los pacientes que iniciaron tratamiento dentro de UTI, el $74 \%$ mejoraron, el $2 \%$ se mantuvo estable y el $24 \%$ fallecieron. En general, de los 100 pacientes tratados, a los 10 días se observó mejoría o estabilización en el $77 \%$ de los pacientes, con fallecimiento del $20 \%$. Los eventos adversos severos fueron tres: dos choques sépticos con defunción y una perforación intestinal manejada con cirugía ${ }^{17}$. Este fármaco, aunque utilizado para arteritis de células gigantes y artritis reumatoide, tiene restricción de no utilizarse por la Food and Drug Administration (FDA) para estas indicaciones con conteos de neutrófilos menores a 2,000 $\mathrm{cel} / \mathrm{mm}^{3}$, de plaquetas menor de $100,000 \mathrm{cel} / \mathrm{mm}^{3}$ o alanina aminotransferasa 0 aspartato aminotransferasa mayor de 1.5 veces del valor superior normal. Este último punto podría resultar limitante, ya que se ha reportado que los pacientes que cursan con cuadro grave de COVID-19 lo hacen con elevación de transaminasas en un 14 a un $59 \%{ }^{18}$.

En el caso del sarilumab, un reporte en prensa reveló resultados preliminares del estudio fase $2 / 3$ de pacientes asignados aleatoriamente a recibir sarilumab $400 \mathrm{mg}, 200 \mathrm{mg}$ o placebo; la población tratada al momento del reporte eran 145 pacientes con dosis de $400 \mathrm{mg}, 136$ con $200 \mathrm{mg}$ y 77 con placebo. Al inicio del estudio los pacientes tenían enfermedad severa en el $28 \%$, enfermedad critica en el $49 \%$ y falla multiorgánica en el $23 \%$. El sarilumab demostró disminuir la PCR en el 79,77 y $21 \%$, respectivamente. La falla al tratamiento, definida por defunción o progresión a ventilación mecánica, fue del 28,46 y $55 \%$ en los grupos de dosis de $400 \mathrm{mg}, 200 \mathrm{mg}$ y placebo respectivamente. Los eventos adversos reportados fueron elevación de enzimas hepáticas de forma reversible o trascendente con raras ocurrencias de neutropenia y trombocitopenia ${ }^{12}$.

El siltuximab también está siendo evaluado con tres registros en clinicaltrials.gov con uno de ellos ya completado, un estudio observacional en pacientes con complicaciones pulmonares severas (NCT04322188), sin embargo, aún no se cuenta con resultados publicados.

\section{Inhibidores de la interleucina 1}

Al igual que con los inhibidores de la IL-6, hasta el momento no hay evidencia que indique o contraindique el uso de inhibidores de la IL-1, en este caso el medicamento anakinra. El sustento de uso de este inhibidor es la efectividad que ha demostrado para detener la tormenta de citocinas en pacientes con tratamiento con células $T$ de receptores de antígenos quiméricas, así como pacientes con síndrome de liberación de mastocitos con falla a inhibidores de la IL-6 $6^{19,20}$. Si bien hay 18 estudios registrados en clinicaltrials.gov con este fármaco, hasta el momento no hay información sobre ensayos clínicos con él más que resultados de series de casos. Se reportó en Francia una serie de nueve casos con neumonía por COVID-19 en hospitalización fuera de UTI con requerimiento de oxígeno $\leq 6 \mathrm{l} / \mathrm{min}$ y $\mathrm{PCR} \geq 50 \mathrm{mg} / \mathrm{l}$ tratados con dosis subcutáneas de anakinra a $100 \mathrm{mg}$ cada $12 \mathrm{~h}$ por 3 días y posteriormente $100 \mathrm{mg}$ cada $24 \mathrm{~h}$ por otros 7 días. Se observó insuficiencia respiratoria en una paciente posterior a $6 \mathrm{~h}$ de la primera dosis, considerándose posible evento adverso, por lo que se suspendió el tratamiento en ella, sin otras reacciones adversas en el resto de los pacientes, observándose en los ocho restantes una respuesta clínica a partir del día 3 respecto a niveles de PCR y oxigenación, con normalización de estos parámetros 
en $5 / 8$ casos al día 11 de tratamiento; los nueve pacientes se mantuvieron vivos ${ }^{21}$.

Otra serie retrospectiva en Milán (Italia) incluyó 29 pacientes con síndrome de insuficiencia respiratoria aguda moderada a severa e hiperinflamación manifestada por $P C R \geq 100 \mathrm{mg} / \mathrm{l}$, ferritina $\geq 900 \mathrm{ng} / \mathrm{ml}$ sin ventilación mecánica invasiva y sin ingreso a UTI en tratamiento con hidroxicloroquina y lopinavir/ritonavir. Se les administró anakinra $5 \mathrm{mg} / \mathrm{kg}$ dos veces al día hasta obtener respuesta clínica, con valoración de efectividad al día 21. Al termino de este tiempo se observó mejoría en PCR y función respiratoria en el $72 \%$ con falla terapéutica por progresión a ventilación mecánica en el 17 y el $10 \%$ de las defunciones. Se reportó bacteriemia en 4/29 pacientes como evento adverso al medicamento ${ }^{22}$.

\section{Inhibidores de JAK}

Las JAK han demostrado efectividad en diversas patologías hematológicas caracterizadas por respuestas inflamatorias desmedidas que resultan en importante lesión tisular de diversos órganos. Uno de los ejemplos más claros de esto es el uso del inhibidor de JAK1/JAK2, ruxolitinib, en enfermedad del injerto contra huésped (EICH). Estudios preclínicos y clínicos han demostrado un rol importante en las señalizaciones por JAK1 y JAK2 en la patogénesis de la EICH. En cultivos celulares de células dendríticas, la inhibición de JAK2 demostró significativamente la supresión de la proliferación de células T citotóxicas con activación y promoción de cambio fenotípico a CD4+ así como linfocitos Treg CD8+CD25+. De la misma manera se observó una inhibición de IL-6 con incremento en el número de células Treg en modelos murinos de EICH intestinal, hepática e incluso pulmonar ${ }^{23}$. Posteriormente los estudios confirmaron clínicamente el grado de respuesta en EICH refractaria a corticosteroides y generaron la autorización de ruxolitinib por la FDA para esta indicación ${ }^{23}$.

Otros datos preclínicos han demostrado que el ruxolitinib bloquea al IFN- $\gamma$ mediante expansión de linfocitos B CD38+ CD27- del centro germinal mientras que el tofacitinib (inhibidor de JAK1/3) inhibe por completo la expansión de células B. En las células presentadoras de antígenos, tanto in vitro como in vivo, los análisis han demostrado que el ruxolitinib inhibe la capacidad de presentación de antígenos mediante inhibición de migración y diferenciación de las células dendríticas con subsecuente reducción del complejo mayor de histocompatibilidad tipo II y con esto la reducción de proliferación de células T con descenso en la producción de citocinas ${ }^{23,24}$.

Con estos datos, la inhibición de la vía JAK representa un blanco atractivo para el tratamiento de patologías caracterizadas por hipercitocinemia, ya que las JAK están expresadas en múltiples linajes de células inmunitarias. Con este conocimiento, se ha direccionado el uso del ruxolitinib en diversas patologías. Otra enfermedad tratada que es un gran ejemplo del potencial en inhibir respuestas inmunitarias desmedidas es el síndrome de linfohistiocitosis hemofagocítica (HLH) primaria observándose respuestas favorables en modelos murinos y casos clínicos de HLH primaria y secundaria reportados por Tefferi, et al. En estos estudios, al igual que como se ha comentado anteriormente, se documentó un descenso en niveles de citocinas proinflamatorias (IL-6, TNF- $\alpha$, GM-CSF, MCP-1 y MIP-1 $\alpha$ ), siendo superior incluso al tratamiento de inhibición solamente de IFN- $\gamma$, esto en los modelos murinos ${ }^{25-28}$.

Otros estudios demuestran efectividad del inhibidor de JAK 1-2 en otras patologías como casos clínicos de tratamiento en mastocitosis sistémica con reducción importante en los niveles de IL-6, TNF- $\alpha$ y MCP-1, e incluso el reporte de tratamientos de dermatomiositis juvenil refractaria con vasculopatía severa posterior a múltiples líneas de tratamiento con remisión y adecuada tolerancia hematológica con respuesta de hasta 14 meses del padecimiento autoinmune 29-31. $^{2}$.

Al ser un medicamento ya conocido en la hematología y en distintas enfermedades autoinmunes, se reportó primeramente su uso en un informe de prensa en pacientes con situación de síndrome agudo respiratorio severo por COVID-19 en la ciudad de Livorno (Italia), presentando respuestas favorables sin defunciones y con adecuada tolerancia en ocho pacientes ${ }^{32}$. Posteriormente se reportó una serie en China de 43 pacientes asignados aleatoriamente a recibir ruxolitinib a dosis de $5 \mathrm{mg}$ cada $12 \mathrm{~h}$ o placebo (vitamina C), ambos brazos junto con el mejor estándar de tratamiento. En este estudio se valoró como objetivo primario el tiempo para la mejoría clínica, definida como disminución de dos puntos en la escala categórica de 7 puntos, encontrándose beneficio a 12 días en el grupo de tratamiento frente a 15 días en el grupo placebo; el $90 \%$ de los pacientes en el brazo de ruxolitinib presentó mejoría en las imágenes de tomografía computarizada de tórax al día 14 comparado con placebo. Se presentaron defunciones en el $14.3 \%$ en el grupo control y no hubo ninguna defunción en el grupo de tratamiento. No hubo diferencias significativas en el 
número de eventos adversos entre ambos grupos ${ }^{33}$. Actualmente se encuentra un protocolo de estudio clínico fase 2 en México coordinado por los autores de la presente revisión registrado en clinicaltrials.gov (NCT04334044) 34

El baricitinib, otro inhibidor de JAK 1-2, se utilizó en Italia en un estudio de casos y controles en donde pacientes 12 fueron tratados con baricitinib a dosis de $4 \mathrm{mg}$ al día por dos semanas. En general se observó que en el grupo tratado con baricitinib, las características de fiebre, saturación periférica de oxígeno, cociente presión alveolar de oxígeno/fracción de oxígeno inspirado, PCR y escala ordinal de síntomas mejoraron tanto a la semana 1 como a la semana 2 comparado con el grupo de control histórico. El traslado a UTI fue necesario en el $33 \%$ de los controles, mientras que ningún paciente en tratamiento con baricitinib requirió ingreso a UTI, con egreso a domicilio en el $8 \%$ en el grupo control y el $58 \%$ en el grupo de tratamiento a las dos semanas. No se reportaron eventos adversos graves en ningún grupo de estudio. Con esto los investigadores concluyeron que el baricitinib resultaba una opción segura para continuar con ensayos clínicos más extensos ${ }^{35}$.

\section{Conclusiones}

Al conocer la base fisiopatológica de esta compleja enfermedad la utilidad del uso de inhibidores de IL es una opción lógica. Los estudios preclínicos y clínicos muestran resultados alentadores al utilizarse en pacientes gravemente enfermos, con especial atención para aquellos medicamentos que inhiben las IL 1 y 6 , pues su papel preponderante en los mecanismos patológicos hasta ahora descritos las ubica como los dos blancos principales de tratamiento.

Si bien los resultados han sido positivos, aún deben explorarse otras alternativas terapéuticas que aseguren un arsenal nutrido y variado de herramientas para el manejo de las diversas presentaciones de esta enfermedad, buscando siempre la prevención de complicaciones graves con la menor tasa de eventos adversos posible.

En hematología tenemos amplia experiencia con el ruxolitinib y conocemos la importante inmunomodulación que produce el ruxolitinib en la cascada de citocinas implicada en las complicaciones pulmonares que genera la infección por SARS-CoV-2, por lo que lo consideramos un medicamento fácil de otorgar y que resultará de alta efectividad en pacientes con síndrome respiratorio agudo severo. Por otra parte, si bien su uso no está tan extendido, el baricitinib promete un rango de efectividad y seguridad muy similar.

\section{Conflicto de intereses}

Los autores declaran no tener conflicto de intereses alguno.

\section{Financiamiento}

Los autores niegan recepción de financiamiento económico relacionado con la publicación de este artículo.

\section{Responsabilidades éticas}

Protección de personas y animales. Los autores declaran que para esta investigación no se han realizado experimentos en seres humanos ni en animales.

Confidencialidad de los datos. Los autores declaran que en este artículo no aparecen datos de pacientes.

Derecho a la privacidad y consentimiento informado. Los autores declaran que en este artículo no aparecen datos de pacientes.

\section{Bibliografía}

1. Cascella M, Rajnik M, Cuomo A, Dulebohn SC, Di Napoli R. Features, evaluation and treatment coronavirus (COVID-19) [Internet]. StatPearls. Treasure Island (FL): StatPearls Publishing; 2020 [consultado: 26/03/2020]. Disponible en: https://pubmed.ncbi.nlm.nih.gov/32150360/

2. World Health Organization. WHO Coronavirus Disease (COVID-19) Dashboard [Internet]. World Health Organization; 2020 [consultado: 21/06/2020]. Disponible en: https://covid19. who.int/?gclid=CjOKCQjwirz3BRD_ARIsAImf7LNUAYB7eNwmPTEf66URxC71RSAElepgdWv2hTGRvyBEvM_JtgbgRngaAhhpEALw_wcB

3. Unidad de Inteligencia Epidemiológica y Sanitaria (UIES) Comunicado Técnico Diario COVID-19 México [Internet]. Gobierno de México, Institución; mayo/2020 [consultado: 20/06/2020]. Disponible en: https://datos. covid-19.conacyt.mx

4. Wu C, Chen X, Cai Y, Xia J, Zhou X, Xu S, et al. Risk factors associated with acute respiratory distress syndrome and death in patients with coronavirus disease 2019 pneumonia in Wuhan, China. JAMA Intern Med. 2020;180(7):934-943.

5. Guo Y-R, Cao Q-D, Hong Z-S, Tan Y-Y, Chen S-D, Jin H-J, et al. The origin, transmission and clinical therapies on coronavirus disease 2019 (COVID-19) outbreak - an update on the status. Mil Med Res. Mil Med Res. 2020;7(1):11

6. Sarzi-Puttini P, Giorgi V, Sirotti S, Marotto D, Ardizzone S, Rizzardini G, et al. COVID-19, cytokines and immunosuppression: what can we learn from severe acute respiratory syndrome? Clin Exp Rheumatol. 2020;38(0):337-42.

7. Huang C, Wang Y, Li X, Ren L, Zhao J, Hu Y, et al. Clinical features of patients infected with 2019 novel coronavirus in Wuhan, China. Lancet. 2020;395(10223):497-506.

8. Channappanavar R, Fehr AR, Vijay R, Mack M, Zhao J, Meyerholz DK, et al. Dysregulated type I interferon and inflammatory monocyte-macrophage responses cause lethal pneumonia in SARS-CoV-infected mice. Cell Host Microbe. 2016;19(2):181-93.

9. Yang X, Cheng X, Tang Y, Qiu X, Wang Z, Fu G, et al. The role of type 1 interferons in Gram-negative bacteria-induced coagulation. Blood. 2020;135(14):1087-100

10. Chen L, Liu HG, Liu W, Liu J, Liu K, Shang J, et al. [Analysis of clinical features of 29 patients with 2019 novel coronavirus pneumonia]. Zhonghua Jie He He Hu Xi Za Zhi. 2020;43(0):E005. 
11. Ziegler CGK, Allon SJ, Nyquist SK, Mbano IM, Miao VN, Tzouanas CN, et al. SARS-CoV-2 receptor ACE2 is an interferon-stimulated gene in human airway epithelial cells and is detected in specific cell subsets across tissues. Cell. 2020;181(5):1016-35.e19.

12. Probarán la droga tocilizumab en el tratamiento de pacientes con coronavirus - Infobae [Internet]. Infobae, Ciencia; 19/03/2020 [consultado: 26/03/2020]. Disponible en: https://www.infobae.com/america/ciencia-america/2020/03/19/roche-anuncio-que-trabaja-en-un-ensayo-clinico-contra-el-coronavirus/

13. Roche inicia un ensayo mundial contra el coronavirus [Internet]. Redacción Médica; 19/03/2020 [consultado: 26/03/2020]. Disponible en: https:// www.redaccionmedica.com/secciones/industria/roche-inicia-un-ensayo-con-tocilizumab-en-hospitalizados-por-coronavirus-6816

14. Coronavirus. Italia pone esperanzas en fármaco para artritis, Tocilizumab [Internet]. El Universal; 18/03/2020 [consultado: 2020 Mar 26]. Disponible en: https://www.eluniversal.com.mx/mundo/coronavirus-italia-pone-esperanzas-en-farmaco-para-artritis-tocilizumab

15. Xu X, Han M, Li T, Sun W, Wang D, Fu B, et al. Effective treatment of severe COVID-19 patients with tocilizumab [Internet]. 2020 [consultado: 2020 Apr 8]. Disponible en: http://chinaxiv.org/abs/202003.00026

16. Toniati P, Piva S, Cattalini M, Garrafa E, Regola F, Castelli F, et al Tocilizumab for the treatment of severe COVID-19 pneumonia with hyperinflammatory syndrome and acute respiratory failure: A single center study of 100 patients in Brescia, Italy. Autoimmun Rev. 2020;19(7):102568

17. Zhang C, Shi L, Wang F-S. Comment Liver injury in COVID-19: management and challenges. Lancet. 2020;5(5):428-30.

18. Regeneron and Sanofi provide update on U.S. phase $2 / 3$ adaptive-designed trial of Kevzara ${ }^{\circledR}$ (sarilumab) in hospitalized COVID-19 patients [Internet]. Regeneron Pharmaceuticals Inc., Regeneron; 2020 [consultado: 22/06/2020]. Disponible en: https://investor.regeneron.com/news-releases/news-release-details/regeneron-and-sanofi-provide-update-us-phase-23-adaptive

19. Shakoory B, Carcillo JA, Chatham WW, Amdur RL, Zhao H, Dinarello CA, et al. Interleukin-1 receptor blockade is associated with reduced mortality in sepsis patients with features of macrophage activation syndrome: Reanalysis of a prior phase III trial. Crit Care Med. 2016;44(2):275-81

20. Monteagudo LA, Boothby A, Gertner E. Continuous intravenous anakinra infusion to calm the cytokine storm in macrophage activation syndrome. ACR Open Rheumatol. 2020;2(5):276-82.

21. Aouba A, Baldolli A, Geffray L, Verdon R, Bergot E, Martin-Silva N, et al. Targeting the inflammatory cascade with anakinra in moderate to severe COVID-19 pneumonia: case series. Ann Rheum Dis. 2020;79(10):1381-2
22. Cavalli G, De Luca G, Campochiaro C, Della-Torre E, Ripa M, Canetti D, et al. Interleukin-1 blockade with high-dose anakinra in patients with COVID-19, acute respiratory distress syndrome, and hyperinflammation: a retrospective cohort study. Lancet Rheumatol. 2020;2(6):e325-e331.

23. Jagasia M, Zeiser R, Arbushites M, Delaite P, Gadbaw B, von Bubnoff N. Ruxolitinib for the treatment of patients with steroid-refractory GVHD: an introduction to the REACH trials. Immunotherapy. 2018;10(5):391-402.

24. T Virtanen A, Haikarainen T, Raivola J Silvennoinen O. Selective JAKinibs: Prospects in inflammatory and autoimmune diseases. BioDrugs. 2019;33(1):15-32.

25. Albeituni S, Verbist KC, Tedrick PE, Tillman H, Picarsic J, Bassett R, et al. Mechanisms of action of ruxolitinib in murine models of hemophagocytic lymphohistiocytosis. Blood. 2019;134(2):147-59.

26. Zinter MS, Herminston ML. Calming the storm in HLH. Blood 2019:134(2):103-4.

27. Sin JH, Zangardi ML. Ruxolitinib for secondary hemophagocytic lymphohistiocytosis: First case report. Hematol Oncol Stem Cell Ther. 2019;12(3):166-70.

28. Slostad J, Hoversten P, Haddox CL, Cisak K, Paludo J, Tefferi A. Ruxolitinib as first-line treatment in secondary hemophagocytic lymphohistiocytosis: A single patient experience. Am J Hematol. 2018;93(2):E47-E49.

29. Fioranelli M, Roccia MG, Lotti T. Treatment of dermatomyositis with ruxolitinib. Dermatol Ther. 2016;29(4):285.

30. Aeschlimann F, Frémond M-L, Duffy D, Rice GI, Charuel J-L, Bondet V, et al. A child with severe juvenile dermatomyositis treated with ruxolitinib. Brain. 2018:141:1-5

31. Hermans MAW, Schrijver B, van Holten-Neelen CCPA, Gerth van Wijk R, van Hagen PM, van Daele PLA, et al. The JAK1/JAK2- inhibitor ruxolitinib inhibits mast cell degranulation and cytokine release. Clin Exp Allergy. 2018;48(11):1412-20.

32. Coronavirus, ospedale di Livorno, primi esiti positivi in pazienti trattati con farmaco "anti-terapie intensive" [Internet]. Città di Livorno [consultado: 01/04/2020]. Disponible en: http://www.comune.livorno.it/articolo/ coronavirus-ospedale-livorno-primi-esiti-positivi-pazienti-trattati-farmaco-anti-terapie

33. Cao Y, Wei J, Zou L, Jiang T, Wang G, Chen L, et al. Ruxolitinib in treatment of severe coronavirus disease 2019 (COVID-19): A multicenter, single-blind, randomized controlled trial. J Allergy Clin Immunol. 2020;146(1):137-46.e3.

34. Ovilla-Martínez R, Cota-Rangel X, de la Peña-Celaya JA, Baez-Islas PE. COVID-19 SARS treatment with ruxolitinib; Ongoing trial and method. Lat Am J Clin Sci Med Technol. 2020;2:83-6.

35. Cantini F, Niccoli L, Matarrese D, Nicastri E, Stobbione P, Goletti D. Baricitinib therapy in COVID-19: A pilot study on safety and clinical impact. J Infect. 2020;16(23):16-23. 